\title{
Artroplastia total de la articulación radiocubital distal
}

\author{
L. R. SCHEKER ${ }^{(1)}$, J. M. BREYER ${ }^{(2)}$

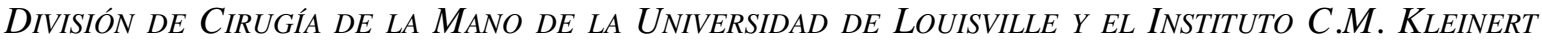 \\ para la Cirugía de la MaNo y Microcirugía. KentuCKY, EStados Unidos \\ ${ }^{(1)}$ PROFESOR ASOCIADO DEL DEPARTAMENTO DE CIRUGÍa \\ ${ }^{(2)}$ FELLOW DE CIRUGÍA DE MANO
}

\author{
Correspondencia: \\ Dr. Luis R. Scheker \\ 225 Abraham Flexner Way, Suite 800 \\ Louisville, Kentucky, Estados Unidos \\ e-mail: 1scheker@kleinertkutz.com
}

\begin{abstract}
La articulación radiocubital distal es un importante eslabón en la transmisión de los movimientos del antebrazo y es la base fundamental para la función en la elevación de objetos. Existen numerosas causas que pueden conducir a una grave lesión de la misma, con importante dolor y limitación funcional. En estos casos la artroplastia total es la única alternativa de tratamiento.

En el presente artículo, se discuten las indicaciones y técnica quirúrgica de la prótesis total de la articulación radiocubital distal, la cual constituye un método efectivo de tratamiento en articulaciones gravemente destruidas. Sólo con la sustitución de todos sus elementos articulares mediante una prótesis autoestable, se devuelve la movilidad a la extremidad, de manera inmediata, estable e indolora.
\end{abstract}

Palabras claves: articulación radiocubital distal, prótesis, artroplastia.
The distal radio-ulnar joint is an important joint that supports the mobility of the forearm and the elevation of objects. There are many causes that lead to severe damage of the joint, in association with severe pain and lost of the function. In these cases of advanced joint destruction, the complete replacement of the joint is the only option of treatment. In this paper we discus the indications and surgical techniques of a distal radio-ulnar joint total prosthesis, which is a very effective treatment of very severe destroyed joints. Only with the replacement of all the structures involved in the distal radio-ulnar joint with a complete self stabilizing prosthesis, the extremity will recover a stable and painless mobility.

Key words: distal radio-ulnar joint, prosthesis, arthroplasty.

Rev. Iberam. Cir. Mano - Vol. 35 • Núm. 2 Noviembre 2007 (7-13)

\section{INTRODUCCIÓN}

$\mathrm{D}$ entro de las patologías causantes de dolor cubital de muñeca, se encuentran aquellas relacionadas con la articulación radiocubital distal (ARCD), pudiendo afectar al componente osteocartilaginoso, al ligamentoso o a ambos. En cuanto al tratamiento se refiere, si bien estas patologías pueden ser tratadas tan- to de forma conservadora como quirúrgica, existen una serie de casos en los que la lesión es tan grave y/o donde han fallado todos los tratamientos quirúrgicos previos, que la única forma de restaurar la función de la extremidad es mediante una artroplastia total radiocubital distal.

El radio y el cúbito se articulan tanto proximal como distalmente mediante una articula- 
ción bicondílea. Ambas articulaciones, otorgan una amplia movilidad al antebrazo, permitiendo al mismo soportar dos tipos principales de fuerzas: cargas axiales (al empuñar o empujar) y fuerzas verticales por el efecto de la gravedad (principalmente al levantar objetos). El primer tipo de fuerzas, se transmiten del carpo al radio, después al cúbito y finalmente al húmero, mientras que las segundas, como es el caso de levantar un objeto con la mano, dependen fundamentalmente del cúbito, el cual actúa como eje de transmisión del antebrazo. Ambas fuerzas actúan de manera combinada en todo momento, siendo el cúbito quien soporta al radio durante la flexión y extensión del codo, permitiendo asimismo la rotación del radio (pronación y supinación) a medida que transmite cargas axiales al húmero ${ }^{1,2}$.

En cuanto a la etiología se refiere, existen múltiples causas que pueden alterar la ARCD; la más frecuente son los traumatismos, donde por ejemplo, la ARCD se ve comprometida en cerca del $30 \%$ de las fracturas de radio distal y en todas las fracturas tipo Essex-Lopresti y Galeazzi. Otras causas son las alteraciones congénitas, artrosis, artritis inflamatoria y neoplasias. El grado de lesión de la ARCD puede ser muy variado, desde una leve artrosis, al extremo de la completa destrucción de la articulación, que se produce al resecar algún segmento del cúbito distal ${ }^{3}$, como ocurre con los procedimientos quirúrgicos de Darrach, Watson, Bower y Sauvé-Kapandji. En estos casos, la alteración biomecánica es máxima, pues se anula el sustento del radio al mismo tiempo que se pierde la adecuada transmisión de fuerzas verticales en el antebrazo. En estas condiciones, se produce un «pellizcamiento cubital», donde el radio sin sustento «cae» e impacta con el cúbito, hecho que se hace muy llamativo al elevar un objeto con la muñeca en posición neutra de pronosupinación ${ }^{4-6}$. Por convención, se utiliza el término «pellizcamiento cubital», en referencia a que el cúbito se mueve contra el radio, cuando en realidad es lo contrario ${ }^{7}$.

La valoración del «pellizcamiento cubital» puede ser constatado clínica y radiográficamente. Sin embargo, hay que tener en cuenta que la simple proyección anteroposterior estática no es capaz de mostrar dicho pellizcamiento, pero sí puede evidenciarse en una radiografía dinámica en la que se reproduzcan las fuerzas verticales que afectan al antebrazo normalmente. En este sentido, dicha radiografía, debe realizarse mientras el paciente sostiene un peso con la mano resistiendo la gravedad (Figura 1).

Cualquiera que sea la causa de la afectación de la ARCD, siempre se deben realizar todos los esfuerzos para lograr una articulación estable y congruente. Así, en casos agudos, se intentará conseguir una reducción ósea lo más anatómica y estable posibles, con una adecuada estabilidad ligamentosa. De comprobarse una consolidación en mala posición, se podrá practicar una osteotomía correctora ${ }^{8,9}$ y si existe una impactación cúbitocarpiana, se puede realizar un acortamiento cubital ${ }^{10}$. El mismo acortamiento puede ser utilizado para la artrosis postraumática y degenerativa incipientes con algún grado de inestabilidad ${ }^{11}$, al cambiar el punto de contacto entre el radio y el cúbito, y mediante el tensado del fibrocartílago triangular (FCT), respectivamente. En casos con inestabilidad sin artrosis, se deberá realizar la reconstrucción de los ligamentos del $\mathrm{FCT}^{12}$.

En aquellos pacientes con grave destrucción articular, cualquiera que sea su causa, probablemente los procedimientos descritos no sean capaces de resolver la patología, de modo que será necesario sustituir la articulación por una prótesis. En estas circunstancias, lo más habitual es que la lesión de la articulación sea generalizada, de modo que una hemiartroplastia, donde sólo se sustituye la cabeza del cúbito, no es sufi-

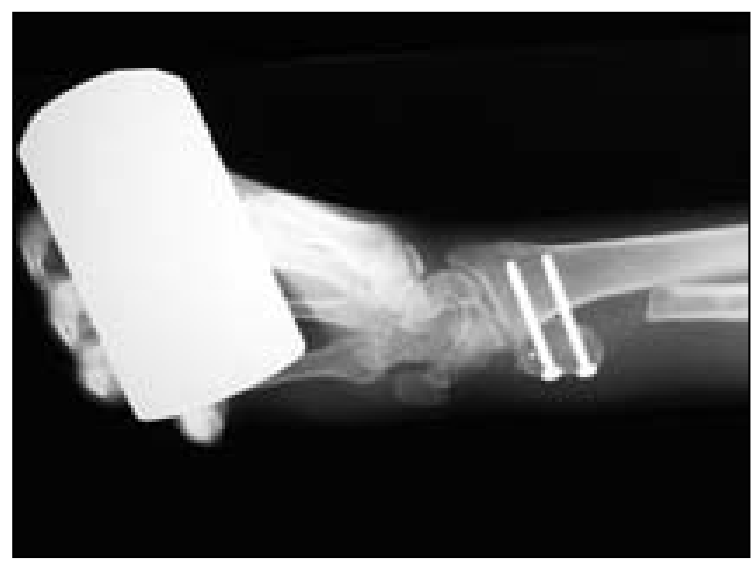

Figura 1: Radiografía dinámica anteroposterior, demostrando el habitual «pellizcamiento cubital» de los pacientes sometidos a una intervención de Sauvé-Kapandji. 
ciente, pues se mantiene el cartílago de la cavidad sigmoidea que generalmente se encuentra dañado y es difícil lograr una adecuada estabilidad de la ARCD. Para estos casos pues, el implante ideal será aquel que sustituya todos los componentes de la articulación (cabeza de cúbito, cavidad sigmoidea y estabilizadores), para que se solucionen los problemas de la articulación de manera completa y definitiva, incluyendo los movimientos de pronación y supinación y la normal transmisión de cargas de la extremidad (axiales y verticales), al mismo tiempo que se consigue su completa estabilidad.

En este artículo se da a conocer la prótesis total de la articulación radiocubital distal APTIS ${ }^{\circledR}$, la cual permite realizar todas las acciones descritas.

\section{CARACTERÍSTICAS DEL IMPLANTE Y APLICACIONES}

La artroplastia APTIS es una prótesis total semiconstreñida no cementada, tipo esfera-cavidad, con una superficie de contacto metal-polietileno (Figura. 2). Al ser una prótesis total puede ser utilizada en pacientes con lesión completa de la articulación o donde se ha resecado parte del cúbito distal. Su fijación se obtiene mediante tornillos en la placa del radio y por medio de la osteointegración, tanto del vástago cubital como del pequeño vástago incorporado a la placa del radio. Al ser semiconstreñida, la prótesis es completamente estable una vez montada y no requiere por lo tanto la conservación y/o cicatrización de los ligamentos o cápsula de la ARCD, que es necesaria y muchas veces difícil de realizar en las hemiartroplastías. Su diseño permite la pronosupinación completa y también el desplazamiento distal del cúbito respecto al radio durante la pronación, en base al contacto entre el polietileno de ultra alta densidad y el metal, lo que confiere una articulación resistente y duradera en el tiempo.

Tal como ha sido comentado, la prótesis de la ARCD APTIS está indicada en pacientes con una lesión avanzada de la ARCD (ósea, cartilaginosa y/o ligamentosa), clínicamente dolorosa y que no ha sido posible resolver mediante tratamientos conservadores y/o reconstructivos.
En este contexto, las indicaciones más frecuentes para su uso son la artrosis (típicamente secundaria a fracturas del radio distal), la lesión del complejo fibrocartílago triangular con inestabilidad y el dolor residual e inestabilidad tras resecciones del cúbito distal (Darrach, SauvéKapandji, etc). Para su utilización se requiere que el paciente sea adulto, con un desarrollo esquelético completo, e idealmente con buena calidad ósea.

La prótesis puede colocarse de forma inmediata, como por ejemplo tras una fractura con grave destrucción articular, o de forma diferida, en cuyo caso es igualmente efectiva. Es frecuente que los pacientes hayan tenido varias cirugías previas a la colocación de la prótesis, en algún caso hasta 14 intervenciones.

En cuanto a las contraindicaciones, la prótesis no puede utilizarse en pacientes con historia de osteomielitis, alergia al níquel, osteoporosis grave o enfermedad sistémica grave. El uso del implante está también contraindicado cuando existe una patología en el hueso, músculos o tendones, que no permita lograr una adecuada fijación, soporte o cobertura de la prótesis.

La artroplastia total APTIS se compone de 4 elementos (Figura 2): 1) placa radial de 5

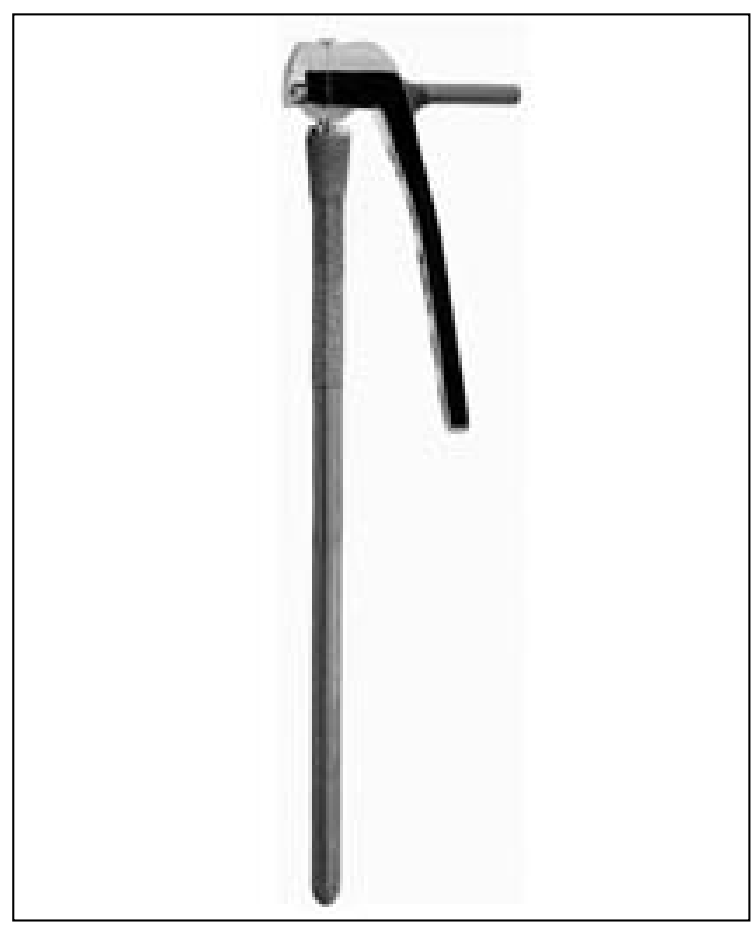

Figura 2: Prótesis total APTIS de la articulación radiocubital distal. 
orificios que posee en su extremo distal un pequeño vástago (lado radial) y una hemiesfera (lado cubital). Dicha placa, disponible en dos tamaños, está premoldeada para ser colocada en la cara dorsocubital del radio, en los 6-7 cm. distales de la cresta interósea. La fijación al radio se logra por medio de su pequeño vástago, que se introduce en dirección cúbitoradial y a través de los 5 orificios de la placa mediante tornillos de $3,5 \mathrm{~mm}$. 2) Cobertor con dos tornillos, el cual va a servir de tapa a la hemiesfera de la placa radial. 3) Cabeza cubital de polietileno de ultra-alta densidad. 4) Vástago cubital de $11 \mathrm{~cm}$. de longitud y con recubrimiento poroso de titanio en su tercio distal para facilitar la incorporación ósea. El vástago está acanalado para permitir una mayor estabilidad rotacional, y es ligeramente cónico para facilitar su inserción. Asimismo, en el extremo distal del vástago, es decir, fuera del cúbito, hay incorporado una clavija, a la cual se encaja la cabeza cubital protésica. El vástago cubital se encuentra disponible en dos diámetros y con distintos largos de cuello, que se utilizarán principalmente en los casos donde no exista o sea necesario resecar una mayor cantidad de cúbito distal. Así pues, la superficie articular de la prótesis está compuesta por la citada cabeza de polietileno de ultra alta densidad, insertada en la clavija del vástago cubital, y la superficie metálica de las hemiesferas de la placa y del cobertor, respectivamente.

\section{TÉCNICA QUIRÚRGICA}

El procedimiento puede realizarse generalmente con un bloqueo axilar y con isquemia de la extremidad mediante manguito neumático. Asimismo, se recomienda el uso de una cobertura plástica yodada impermeable de toda la extremidad para reducir el contacto con la piel y minimizar así el riesgo de infecciones. El antebrazo se coloca en pronación completa. Mediante una incisión dorsocubital de 7 a $8 \mathrm{~cm}$. y angulada distalmente en forma de palo de golf (Figura 3), se aborda la articulación entre el quinto y sexto compartimiento, es decir, entre el tendón del extensor digiti minimi (EDM) y el extensor carpi ulnaris (ECU), aunque pue-

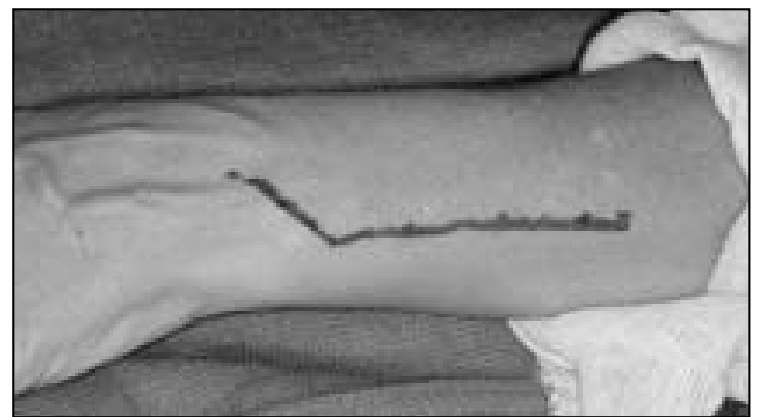

Figura 3: La incisión en palo de golf se localiza en el dorso de la muñeca, entre el extensor carpi ulnaris y el extensor digiti minimi.

de utilizarse también el intervalo entre el ECU y el flexor carpi ulnaris (FCU). El colgajo del retináculo extensor debe disecarse en una extensión de 3 a $4 \mathrm{~cm}$., con el fin de utilizarlo finalmente para cubrir la prótesis y separarla de los tendones. La vaina del ECU debe liberarse completamente para prevenir futuras compresiones. A continuación, se expone la superficie dorsal del cúbito así como el segmento dorsocubital del radio. Para la resección de la cabeza cubital debe realizarse, con sierra eléctrica, un corte inmediatamente proximal a la misma. Una vez resecada se facilita la disección del radio, al mismo tiempo que permite la exéresis de todos los osteofitos que pudieran encontrarse alrededor de la ARCD. Seguidamente, se resecan los 8 a $9 \mathrm{~cm}$. distales de la membrana interósea, con el fin de exponer la cresta radial, donde irá colocada la placa. De encontrarse aun presente el FCT, se debe conservar su inserción radial, ya que actuará como una barrera entre la prótesis y el carpo. Una vez expuesto el radio, se coloca la placa de prueba sobre la cresta del radio, con el borde palmar de la placa en el mismo plano de la cortical volar del radio y con la parte distal de la placa en la zona de la cavidad sigmoidea, debiendo tener como mínimo $3 \mathrm{~mm}$. de espacio entre el extremo distal de la placa y la superficie articular del radio. Se puede fresar el borde distal del radio para mejorar el contacto de la placa con el radio. Teniendo una posición adecuada controlada bajo escopia, se coloca un tornillo en el orificio oval de la placa y se perfora a través del orificio distal de la placa, donde posteriormente se introducirá el pequeño vástago de la placa del radio. Confirmada la posición, se colocará la 


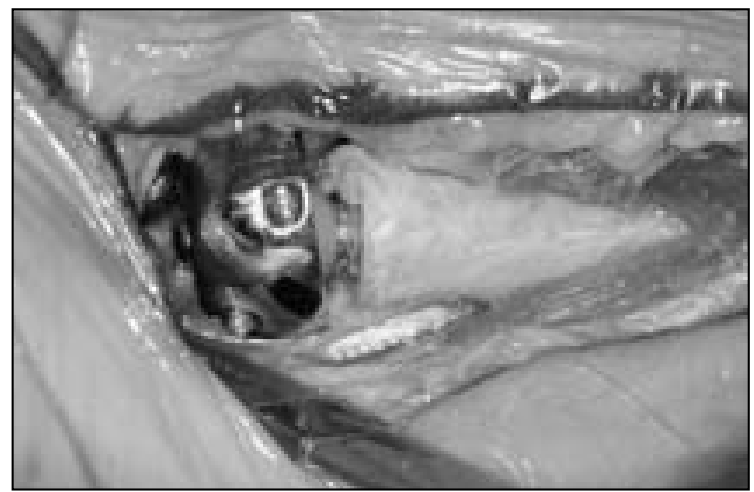

Figura 4: La colocación del cobertor con tornillos, estabiliza la nueva articulación.

placa definitiva, introduciendo su propio vástago en el radio y fijándola con 5 tornillos de $3,5 \mathrm{~mm}$. A continuación, se define la longitud real del cúbito necesario, mediante un medidor y su relación con la placa del radio, teniendo en consideración que la osteotomía del cúbito se realice sobre hueso de buena calidad. En los casos donde exista una pérdida ósea considerable, generalmente por cirugías previas (Darrach, Sauvé-Kapandji), se deberá utilizar el vástago extendido, el cual se determinará previamente en la radiografía y se confirmará en el campo quirúrgico y por escopia. Después de la resección del cúbito distal, se prepara el canal con la fresa correspondiente al tamaño de la prótesis a utilizar. A continuación, y después de irrigar copiosamente el canal medular, se introduce e impacta el vástago y se ensamblan los componentes de la prótesis, colocando la cabeza cubital en la clavija correspondiente a su vástago y, seguidamente, en la hemiesfera correspondiente a la placa del radio. El cobertor metálico, que se fija a dicha hemiesfera con dos tornillos, completa el ensamblaje (Figura 4), que se deberá controlar bajo escopia (Figura 5). Se asegurará la libre y completa movilidad de la articulación en todo el rango de prono-supinación, ya que de no ser así, se deberán eliminar los posibles puntos de contacto entre el cúbito y la placa del radio, y evaluar la necesidad de liberar aún más la membrana interósea.

Finalmente, el colgajo del retináculo extensor se utiliza para cubrir la prótesis y separarla de los tendones. Se recomienda liberar el torniquete antes del cierre de la herida e irrigar la herida para minimizar la aparición de osifica-

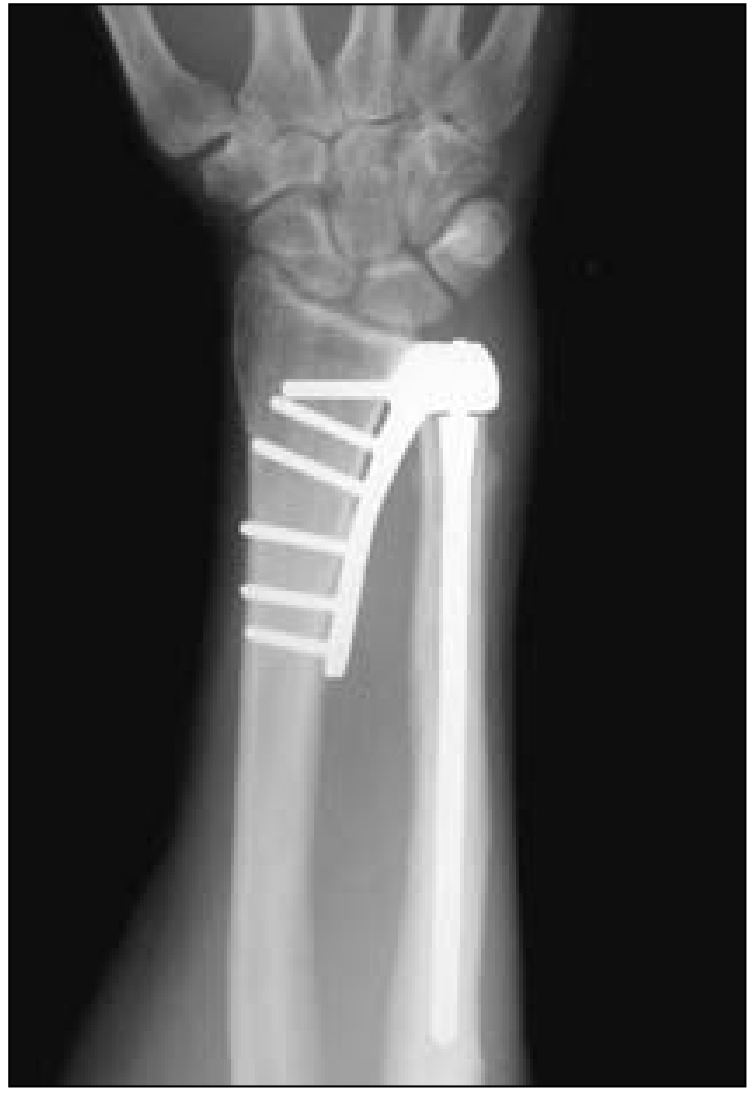

Figura 5: Visión radiográfica de la prótesis.

ciones heterotópicas. Debido a la estabilidad intrínseca e inmediata de la prótesis, no existe riesgo de luxación, por lo que no requiere una inmovilización rígida, sólo se coloca un vendaje compresivo abultado y se inicia la movilidad inmediata.

\section{FALLOS POTENCIALES}

El uso de radiografías sin escala de medida, puede causar error en la selección del tamaño de la prótesis, especialmente en la elección del vástago cubital. En este sentido, si es muy pequeño, el vástago no se ajustará con lo que se aflojará posteriormente, mientras que si es muy grande, no se logrará su completa introducción, corriendo el riesgo de provocar una fractura. Asimismo, si se impacta demasiado dentro de su canal medular, existe el riego de una inadecuada relación entre la cabeza cubital y la esfera del radio, y, en consecuencia, a un excesivo roce del polietileno, lo que ocasionará su desgaste. Sin embargo, de existir un vástago de- 
masiado impactado, es preferible la traslación proximal de la placa radial que realizar la tracción del vástago cubital, ya que el diseño «press-fit» de la prótesis, impide este gesto. Por lo que respecta a la placa del radio, si es demasiado grande, su vástago distal puede protruir por la cortical radial.

Otro aspecto a tener en cuenta es la inadecuada cobertura de la prótesis para separarla de los tendones extensores, pues puede ser motivo de irritación y desgaste de los mismos, hecho que puede ocurrir también cuando se colocan tornillos demasiado prominentes.

\section{RESULTADOS}

El objetivo fundamental de la prótesis APTIS es la eliminación del dolor y permitir, a una persona con una articulación destruida, recobrar su función y, por lo tanto, la de toda la extremidad. Con la experiencia adquirida con más de 200 pacientes, hemos observado que tras la artroplastia, los pacientes recuperan rápidamente el movimiento de pronosupinación y, muy importante, sin dolor. Con respecto a la función, un elemento a destacar, pero que no se analiza con frecuencia en este tipo de procedimientos, es que los pacientes recuperan la capacidad de soportar y elevar cargas verticales, debido a la estabilidad de la nueva articulación, lo que en la práctica permite al paciente utilizar libremente la extremidad. Hasta la fecha, no hemos podido constatar ningún aflojamiento aséptico, ni ha sido necesario cambiar la cabeza cubital de polietileno. Sin embargo, se han producido 2 casos de fractura del vástago cubital, aunque ambos debidos a traumatismos de alta energía en accidentes de tráfico. En ambos casos se procedió a su revisión y sustitución del vástago, sin alteración de la función articular. En 2 casos se produjo una infección, siendo necesario retirar la prótesis. En uno de estos casos, antes de la colocación de una nueva prótesis, requirió la introducción de un rosario de antibióticos y se colocó una nueva prótesis seis meses después. En 4 casos se produjo osificación heterotópica que limitó la movilidad. Sin embargo, la resección de la misma solucionó el problema, sin que hasta el momento se haya producido su recidiva.

En los casos iniciales donde no se colocó el colgajo del retináculo extensor sobre la prótesis, se produjo en algunos casos una tendinitis del ECU, la cual, en caso de ser persistente, requiere su liberación.

\section{CONCLUSIONES}

La articulación radiocubital distal es una importante articulación que permite la movilidad al antebrazo y es la base fundamental para la función en la elevación de objetos. En los casos de grave destrucción, la prótesis total autoestable puede ser el único procedimiento que permita devolver al paciente una articulación funcional e indolora. 


\section{BIBLIOGRAFÍA}

1. Hagert CG. The distal radioulnar joint. Hand Clin, 1987; 3: 4150.

2. Hagert CG. The distal radioulnar joint in relation to the whole forearm. Clin Orthop, 1992; 275: 56-64.

3. Bieber EJ, Linscheid RL, Dobyns $\mathrm{JH}$, et al. Failed distal ulna resections. J Hand Surg A, 1988; 13: 193-200.

4. Shaaban H, Giakas G, Bolton M, et al. The distal radioulnar joint as a load-bearing mechanism-a biomechanical study. J Hand Surg A, 2004; 29: 85-95.

5. Shaaban H, Giakas G, Bolton M, et al. The load-bearing characteristics of the forearm: pattern of axial and bending force transmitted through ulna and radius. J Hand Surg B, 2006; 31: 274-9.

6. Shaaban H, Giakas G, Bolton M et al.: Contact area inside the distal radioulnar joint: effect of axial loading and position of the forearm. Clin Biomech, 2007; 22: 313-8.

7. Lees VC, Scheker LR. The radiological demonstration of dynamic ulnar impingement. J Hand Surg B, 1997; 22: 448-50.

8. Wagner $\mathrm{H}$. [Proceedings: Corrective surgery after lesions of the distal end of the radius (author's transl)] Langenbecks Arch Chir, 1973; 334: 211-9.
9. Viegas SF. A new modification of corrective osteotomy for treatment of distal radius malunion. Tech Hand Up Extrem Surg, 2006; 10: 224-30.

10. Rayhack JM. Ulnar shortening. Tech Hand Up Extrem Surg, 2003; 7: 52-60.

11. Scheker LR, Severo A. Ulnar shortening for the treatment of early post-traumatic osteoarthritis at the distal radioulnar joint. J Hand Surg B, 2001; 26: 41-4.

12. Scheker LR, Belliappa PP, Acosta R. Reconstruction of the dorsal ligament of the triangular fibrocartilage complex. J Hand Surg B, 1994; 19: 310-8. 